\title{
Finite Element Simulation of Welding of Large Components Based on Inherent Strain and Thermo Elastic Plasticity
}

\author{
FANG Yuanbin 1, 2, a , ZONG Xuemei, 2, b and WANG Can 1, 2, c \\ ${ }^{1}$ Jiangsu Xuzhou Engineering Machinery Research Institute, Jiangsu Xuzhou 221004, China \\ ${ }^{2}$ State key Laboratory of Intelligent Manufacturing of Advanced Construction Machinery, XCMG \\ Construction Machinery Co., Ltd, Jiangsu Xuzhou 221004, China \\ afybflying@163.com, bongxuemei@139.com, ${ }^{c} 18251591969 @ 163 . c o m$
}

Keywords: Inherent strain; thermo elastic plastic; welding deformation; stress

Abstract. Based on inherent strain theory, it predicts and optimizes welding deformation of movable arm by means of welding numerical simulation. At the same time, based on thermal elastic-plastic theory, it predicts stress distribution, and finite element model is verified correctly by experiments. The results show that maximum deformation occurs at the front ear plate, and maximum unilateral deformation is $1.95 \mathrm{~mm}$. Compared with experimental results, error is about $11 \%$, which proves the accuracy of simulation results. The best welding sequence is $1,5,4,2,3$. Maximum deformation of front ear plate is reduced by $0.47 \mathrm{~mm}$, and welding deformation is reduced by $24.1 \%$. Maximum stress is located at the intersection of reinforcing plate and lower cover, and peak value is $289.4 \mathrm{MPa}$. Simulation results are in good agreement with experimental results, and error is controlled within $11.96 \%$, which verifies the validity of finite element model.

\section{Introduction}

With the continuous improvement of intelligent manufacturing capability and precision in the field of engineering machinery, traditional welding process design based on experience of technical personnel cannot meet the requirements of engineering application [1].With the development of welding numerical simulation technology, it provides a scientific tool for the prediction of welding deformation and stress. In order to ensure the accuracy of calculation, it usually analyzes based on the thermal elastic plastic theory [2]. But welded seams are usually very much, and grids are often at the level of millions. At the same time, it has a large amount of calculation, highly nonlinear and difficult to converge. With high-performance computers, computing time is up to one month, and far beyond the requirements of Engineering applications. In order to solve the problem of computational efficiency, Japanese scholars put forward the inherent strain theory [3] which greatly simplifies welding simulation process. It thus greatly improves computational efficiency, but simulation results are difficult to ensure accuracy.

Experts and scholars at domestic and foreign have done a lot of research on the basis of the theory of thermo elastic plastic and inherent strain. J. H. Wang and H. Lu, Professors of Shanghai Jiao Tong University [4], applied the inherent strain theory to predict welding deformation of large structures, such as large hull. D. Deng, professor of Chongqing University and Murakawa, professor of Osaka University [5], used the inherent strain theory to predict the deformation of sheet metal. A. L. Lu and Z. P. Cai, Professors of Tsinghua University [6], used similitude principles based on the method of elastic strain energy to analyze accurately calculation precision of large structure welding deformation. Welding numerical simulation technology has been improved in the engineering application. Empirical methods are gradually accumulated, such as geometric model symmetry, grids transition division, parallel computing and so on.

In order to meet the requirements of precision and efficiency of engineering application, research makes use of inherent strain theory calculation efficiency, and designs full factorial experiments to predict the optimum welding sequence. With high accuracy of thermal elastic plastic theory, it accurately predicts stress distribution of large components movable arm. 


\section{Establish the finite element model}

Test preparation. Material of movable arm is Q345A. The distance between Front ear plate and pinhole center is $3000 \mathrm{~mm}$. The thickness of upper and under ear plate is $20 \mathrm{~mm}$. The thickness of other plates is $8 \mathrm{~mm}$. The model is shown in Fig. 1. It is welded by Fronius 500 welding machine, and welding wire is ER50-6. The diameter of welding wire is $1.2 \mathrm{~mm}$, and the groove angle is 60 degrees. Welding parameters are shown in table 1.

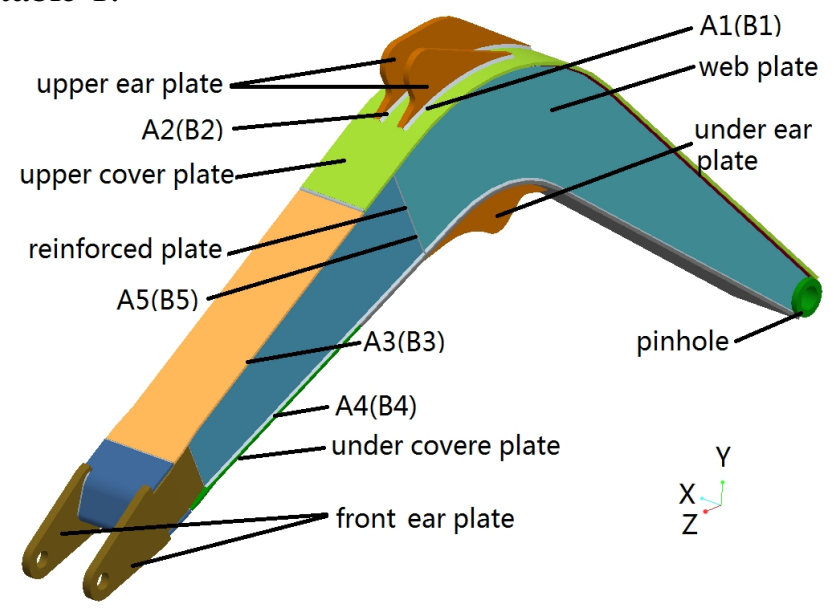

Fig. 1 The solid model of movable arm Table 1 Welding parameters

\begin{tabular}{c|c|c}
\hline Layers & The first layer & The second layer \\
\hline Arc voltage [V] & $26-28$ & $32-34$ \\
\hline Arc current [A] & $240-260$ & $280-320$ \\
\hline Welding speed [mm/s] & 24 & 15 \\
\hline
\end{tabular}

As shown in Figure 1, weld seams between upper ear plate and upper cover plate are respectively defined as A1, A2. Correspondingly, weld seams between under ear plate and under cover plate are respectively defined as B1, B2. Weld seam between upper cover plate and web plate is defined as A3, and symmetrical weld is defined as B3. Weld seam between under cover plate and web plate is defined as A4, and symmetrical weld is defined as B4. Weld seam between web plate and reinforced plate is A5, and symmetrical weld is defined as B5.

Establishment of grid model. The size of movable arm is large. In order to effectively control calculation model based on the thermal elastic-plastic theory, it adopts the transition grid. It deals with dense grid in the weld area and heat-affected zone, and large grid away from weld seams position. The minimum size of grid is $0.5 \mathrm{~mm}$, and the maximum size of grid is $37.5 \mathrm{~mm}$. The total number of grid unit is 175767 , and the number of node is 285284 . It is shown in Fig. 2 (a). A large amount of time is needed for manual generation, so the model adopts shell element based on the inherent strain theory. The model uses topology segmentation and automatic meshing to refine meshing near the weld zone. Grid is sparse away from weld seams. The minimum size of grid is $0.5 \mathrm{~mm}$, and the maximum size of grid is $15 \mathrm{~mm}$. The total number of grid unit is 231480 , and the number of node is 226689 . It is shown in Fig. 2 (b).

Establishment of material parameters. Thermal physical and mechanics parameters of material change with temperature. The material parameters affect accuracy of simulation results. Simulation is used for materials of Q345A.

Heat source model checking. It can effectively simulate transient thermal loading based on the thermo elastic plastic theory. Establish the proper heat source model reflects carbon-dioxide arc welding [7]. Double ellipsoid heat source fully considers the characteristic of heat source that temperature of front heat source changes steeply, and temperature of rear heat source changes slowly in the welding process. Ignoring the flow of welding molten pool, it is defined as quasi steady state. 
According to the shape of heat resource, weld prefabricated grids are filled. Elements based on life-death technique are used to activate.

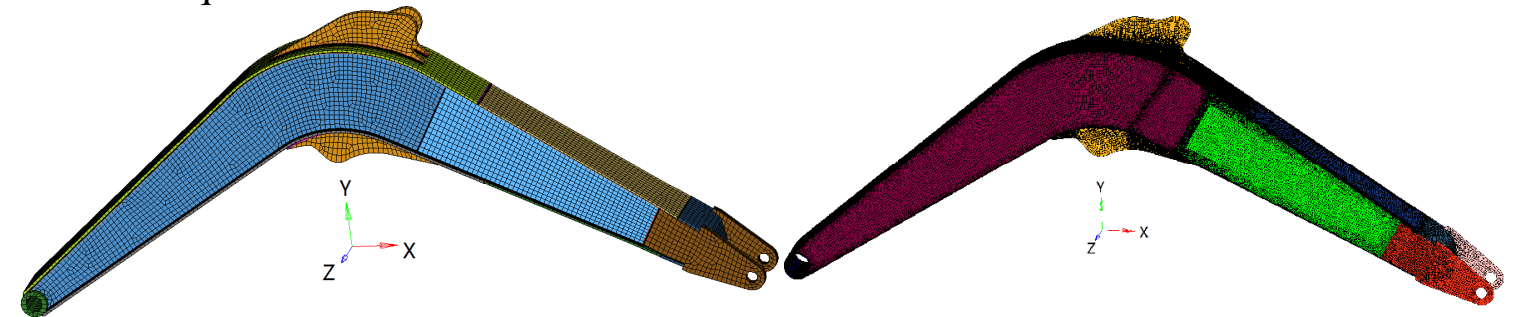

(a) Mesh model based on thermo elastic plastic theory (b) Mesh model based on inherent strain theory Fig. 2 Mesh model

According to the design requirements of welded joints, three kinds of joints which numbered 1-5 are tested respectively. It obtains test specimen, and measures welding high, bead width, penetration depth and heat affected zone. Macro morphology of heat source is shown in Fig. 3.

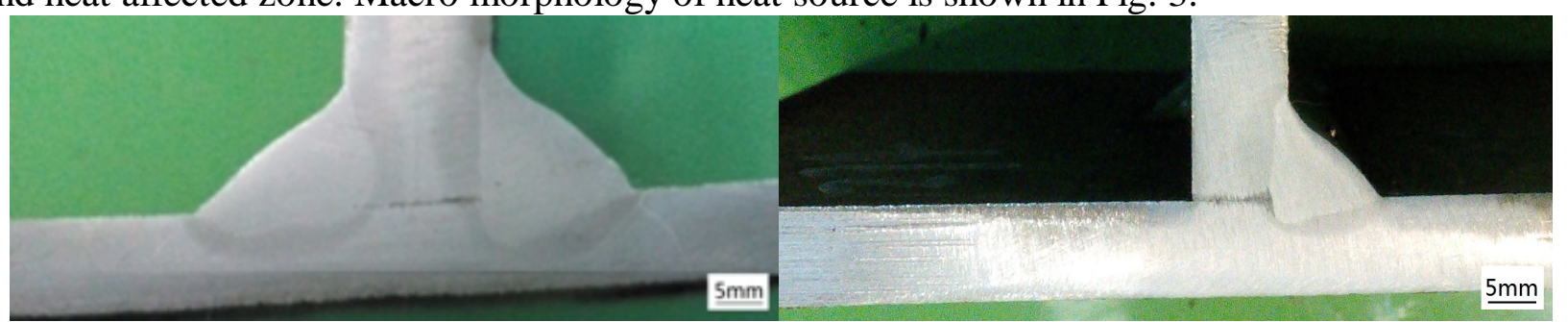

(a)Heat resource of experiment numbered 1and 2 (b)Heat resource of experiment numbered 3 and 4

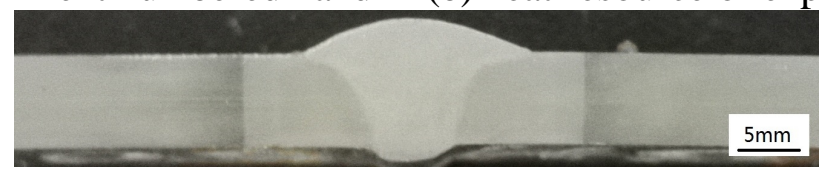

(c)Heat resource of experiment numbered 5

Fig. 3 Heat resource

Boundary condition. Mechanical boundary conditions are set up by adding displacement constraint. Considering the fixture in circumferential position of front ear plate fixed in the hole into the machine, it is definition of constraints to restrict $\mathrm{X}, \mathrm{Y}$ and $\mathrm{Z}$ circumferential direction to simulate rigid fixture. It is definition of constraints to restrict $\mathrm{Y}$ and $\mathrm{Z}$ displacement to simulate the pin hole, and does not affect longitudinal shrinkage deformation of front ear plate along the direction of hole.

Schemes of welding sequence. Symmetric welding can effectively control deformation. Welding sequence scheme adopts the symmetrical welding principle. Symmetrical positions of A and B are divided into a group. It is designed of full factorial experiments on weld seams numbered 1-5. Each welding sequence test factor cannot be repeated, and each scheme contains 5 factors. It is shown in Eq. 1.

$$
\mathrm{y}=\mathrm{a} * \mathrm{~b} * \mathrm{c} * \mathrm{~d} * \mathrm{e}
$$

In the formula, $\mathrm{y}$ is total number of schemes; $\mathrm{a}, \mathrm{b}, \mathrm{c}, \mathrm{d}$, e, respectively, is number of test factors. According to different orders of test factors, it works out 120 schemes of welding sequence.

\section{Simulation results analysis and experimental verification}

Welding deformation simulation results analysis and verification. The original welding sequence is $1,2,3,4,5$. It is obtained contour of welding deformation based on the inherent strain theory. It is shown in Fig. 4 (a). It shows that the position of maximum deformation occurs in the front ear plate, and maximum unilateral deformation is $1.95 \mathrm{~mm}$. From theoretical analysis, upper cover plate, under cover plate and web plate of movable arm are of high stiffness. Upper ear plate, lower ear plate and front ear plate are of low stiffness. The position of maximum deformation is reasonable [12]. According to accounting welding sequence scheme, they are obtained results of welding deformation. 
Through comparative analysis, welding sequence 1, 5, 4, 2, 3 is best, and welding deformation of front ear plate is least. It is shown in Fig. 4 (b). Through comparison of Fig. 4, it shows that maximum deformation of front ear plate is reduced by $0.47 \mathrm{~mm}$, and welding deformation is reduced by $24.1 \%$.
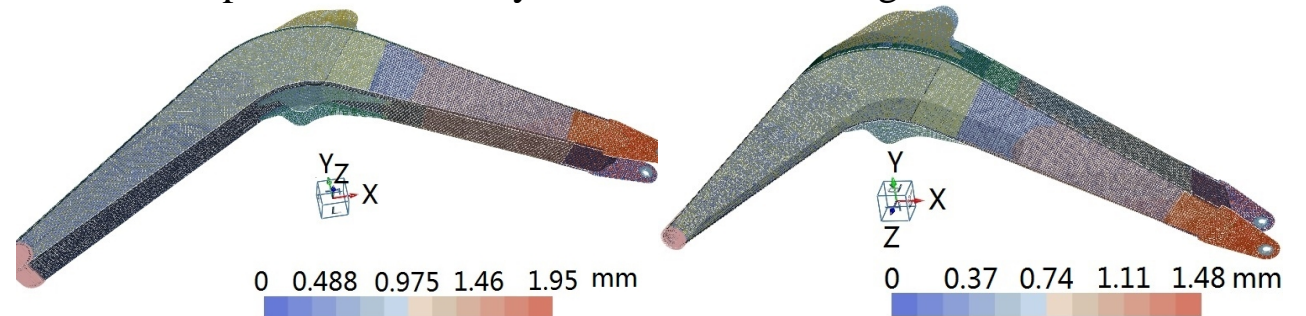

(a)Contour of original welding deformation (b)Contour of optimal welding deformation

Fig. 4 Contour of welding deformation

The distance between two front ear plates is measured by a height gauge on the welding platform. The designed space is $184 \mathrm{~mm}$. It selected randomly three groups of movable arm to verify. It takes an average value as experimental deformation. They are three groups in total. It is shown in table 2.

Table 2 Several welding schemes

\begin{tabular}{c|c|c|c}
\hline Test & Before welding [mm] & After welding [mm] & difference value[mm] \\
\hline 1 & 184.25 & 179.75 & 4.50 \\
\hline 2 & 184.40 & 180.25 & 4.15 \\
\hline 3 & 184.20 & 179.85 & 4.35 \\
\hline
\end{tabular}

According to Table 2, we can see that the average value of welding deformation is $4.33 \mathrm{~mm}$, and unilateral deformation is $2.165 \mathrm{~mm}$. Compared with simulation results, error is about $11 \%$, which meets requirements of engineering application.

Welding stress simulation results analysis and verification. On the basis of welding deformation prediction to meet the requirements of engineering application, welding stress is accurately predicted. In order to make research more practical, it analyzes the stress concentration factor position of movable arm. It is shown in Fig. 5 (a). Based on the thermal elastic plastic theory, it simulates welding process of movable arm, and obtains stress distribution. It is shown in Fig. 5 (b). It shows that the maximum stress is located at the intersection of reinforcing plate and under cover plate, and peak stress is $289.4 \mathrm{MPa}$. Stress concentration factor occurs at the location of the crossover. It is formation of a certain torque at the crossing position caused by weld shrinkage stress of ear plate welding. It increases the position of tension stress, so the position occurs stress concentration factor, and residual stress is max.

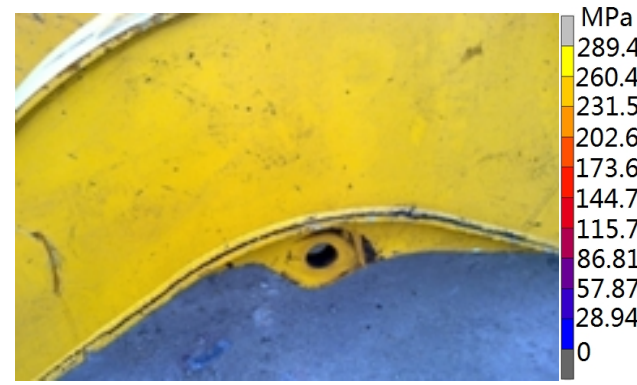

(a)Movable arm cracking

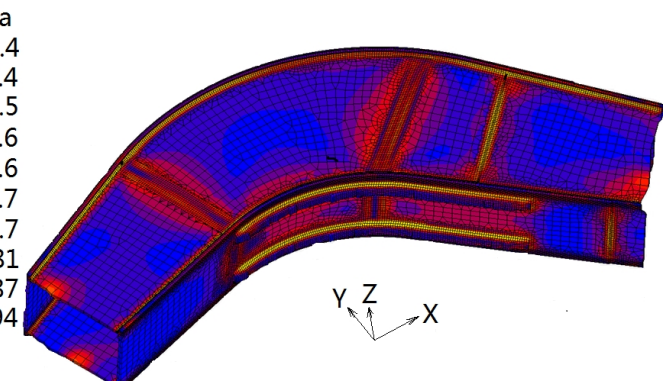

(b)Welding stress distribution

Fig. 5 Movable arm cracking and contour of welding stress

Test verification. It measures stress near the toe of weld seam A5 by HK21A type stress analyzer. Arc position is located at the cross position between upper cover plate and reinforcing plate. The distance away from arc position is $55 \mathrm{~mm}, 102 \mathrm{~mm}, 149 \mathrm{~mm}, 216 \mathrm{~mm}, 273 \mathrm{~mm}$. Points measured are as close as possible to weld toe. Simulation results are compared with test results. It is shown in Fig. 6.

It indicates that stress results are in good agreement with test results, and error is controlled within $11.96 \%$. It verifies the validity of finite element model established. The accuracy of stress prediction meets the requirements of engineering applications. 


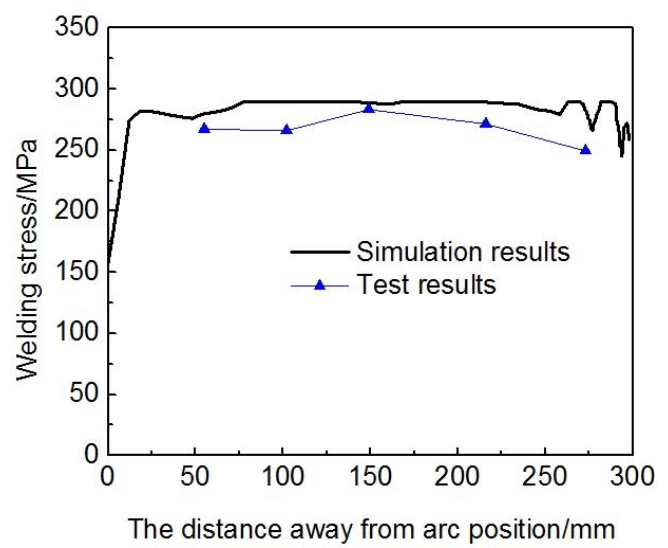

Fig. 6 Experiment measurement and verification

\section{Conclusions}

Considering the balance of calculation efficiency and precision, it predicts welding deformation and stress of large components based on different theories. Based on the inherent strain theory, it predicts welding deformation of movable arm. Based on the thermal elastic plastic theory, it predicts welding stress of movable arm. Based on the inherent strain theory, it obtains maximum deformation occuring at the front ear plate. Compared with experimental results, error is about $11 \%$, which proves the accuracy of simulation results. At the same time, it quickly predicts the best welding sequence. Maximum deformation of front ear plate is reduced by $24.1 \%$. Based on the thermo elastic plastic theory, it obtains maximum stress located at the intersection of reinforcing plate and lower cover, and the peak value is $289.4 \mathrm{MPa}$. Simulation results are in good agreement with experimental results, and error is controlled within $11.96 \%$, which verifies the validity of finite element model. Make full use of the advantages of two theories, they effectively apply in the engineering practice, and solve the problem of computational efficiency and accuracy. However, it is still necessary to research grid adaptive technology and the heat source parameter database of large components.

\section{Acknowledgements}

This work was financially supported by the Jiangsu Natural Science Foundation (BK20140229), and National Science and Technology Support Program (2015BAF07B02).

\section{References}

[1] X. T. Tian: Welding Structure (China Machine Press Publications, China 1982).

[2] C. S. Wu, H. LU, and Y. H. Wei: Welding \& Joining Vol. 1 (2012), p. 10-22.

[3] Y. Ueda, Y. C. Kim, and M. G. Yuan: J Trans. of JWRI, Vol. 18, No. 1 (1989), p. 135-141.

[4] J. H.WANG, H. LU, L. and W. WEI: Chinese Journal of Mechanical Engineering, Vol. 23, No. 6 (2002), p. 36-40.

[5] D. Deng, H. Murakawa and N. Ma: Science and Technology of Welding and Joining, Vol. 17, No. 1 (2012), p. 13-21.

[6] Z. P. Cai, H. Y. Zhao, A. L. Lu, D. B. Liu and Y. Wang: Chinese Journal of Mechanical Engineering, Vol. 38, No. 10 (2002), p. 100-104.

[7] Y. B. Fang, X. M. Zong, H. Q. Zhang, B. Sun and X. Q. Yin: Welding Technology, Vol. 45, No. 3 (2016), p. 5-8. 